\title{
Philosophiques
}

\section{Perception et culture chez Merleau-Ponty}

\section{Marcus Sacrini A. Ferraz}

Volume 35, numéro 2, automne 2008

URI : https://id.erudit.org/iderudit/000411ar

DOI : https://doi.org/10.7202/000411ar

Aller au sommaire du numéro

Éditeur(s)

Société de philosophie du Québec

ISSN

0316-2923 (imprimé)

1492-1391 (numérique)

Découvrir la revue

Citer cet article

Ferraz, M. S. A. (2008). Perception et culture chez Merleau-Ponty. Philosophiques, 35(2), 297-316. https://doi.org/10.7202/000411ar

\section{Résumé de l'article}

L'auteur essaie de montrer que le paradigme anthropologique de l'incarnation, présenté par Thomas Csordas sous l'inspiration de Merleau-Ponty, entre autres, ne trouve pas de soutien dans la Phénoménologie de la perception, mais dans les textes intermédiaires de Merleau-Ponty (sections 1-5). En plus, l'auteur propose que l'idée de perception informée culturellement, contenue dans ces textes, permet d'assouplir l'opposition entre conceptualistes et non-conceptualistes dans le débat contemporain sur la perception (sections $6-7)$. d'utilisation que vous pouvez consulter en ligne.

https://apropos.erudit.org/fr/usagers/politique-dutilisation/ 


\title{
Perception et culture chez Merleau-Ponty
}

\author{
MARCUS SACRINI A. FERRAZ \\ Université de São Paulo - Brésil \\ sacrini@usp.br
}

\begin{abstract}
RÉSUMÉ - L'auteur essaie de montrer que le paradigme anthropologique de l'incarnation, présenté par Thomas Csordas sous l'inspiration de Merleau-Ponty, entre autres, ne trouve pas de soutien dans la Phénoménologie de la perception, mais dans les textes intermédiaires de Merleau-Ponty (sections 1-5). En plus, l'auteur propose que l'idée de perception informée culturellement, contenue dans ces textes, permet d'assouplir l'opposition entre conceptualistes et non-conceptualistes dans le débat contemporain sur la perception (sections 6-7).
\end{abstract}

\begin{abstract}
The author tries to show that the anthropological paradigm of embodiment, put forward by Thomas Csordas, who drew his inspiration from Merleau-Ponty among others, is not supported by Phenomenology of Perception but by the intermediate texts from Merleau-Ponty (sections 1-5). In addition, the author proposes that the idea of culturally informed perception, comprised in such texts, allows us to minimize the opposition between conceptualists and non conceptualists in the contemporary debate on perception
\end{abstract}

\section{Le préobjectif et l'anthropologie}

Par l'idée d'expérience préobjective développée dans la Phénoménologie de la perception (publiée en 1945), Merleau-Ponty entend circonscrire un champ de phénomènes toujours lié à l'activité perceptive et motrice. Ces phénomènes ne peuvent pas se décrire par des concepts ou des mesures rigides, qui sont en général appliqués à des objets étudiés scientifiquement. C'est pourquoi le champ en question doit être dit préobjectif: il s'agit d'un domaine où règne l'indétermination et l'ambiguiité, caractéristiques qui ne sont pas étudiées par des recherches objectives. Pour dévoiler ce champ phénoménal, Merleau-Ponty reprend la réduction phénoménologique husserlienne, c'est-à-dire qu'il déconsidère le savoir sur le monde objectif pour n'étudier que les vécus subjectifs. Toutefois, la réduction qu'opère Merleau-Ponty ne l'amène pas jusqu'au sujet transcendantal pur, tel que proposé par Husserl dans plusieurs livres, mais elle rend explicite l'organisation spontanée des objets perçus selon les structures de la perception concrète.

Thomas Csordas prétend instaurer un nouveau paradigme d'investigation anthropologique, et dans ce but il utilise, parmi d'autres références philosophiques, cette explicitation merleau-pontyenne de l'organisation de l'expérience. Selon Csordas, à l'intérieur de ce paradigme (qui est appelé paradigme de l'incarnation) il s'agit de chercher "comment les objets culturels (y compris les soi [selves]) sont constitués ou objectifiés [objectified] non pas dans le processus d'ontogenèse et de socialisation infantile, mais [...] dans le flux de 
la vie culturelle adulte ${ }^{1} »$. Ainsi, de même que Merleau-Ponty essaie de dévoiler la présentation des objets par des phénomènes perçus, Csordas propose de montrer comment les formations culturelles sont sédimentées par des activités subjectives. Nous essaierons de montrer que cette idée de Csordas peut en effet s'appuyer sur quelques conceptions de Merleau-Ponty, mais non sur celles de la Phénoménologie de la perception. Nous verrons que dans ce livre il n'y a pas de légitimation théorique d'une investigation anthropologique, c.-à-d., d'un recueil de l'organisation du milieu humain selon la diversité culturelle. La considération de la diversité culturelle n'aura lieu que dans la période intermédiaire de l'œuvre de Merleau-Ponty. Nous verrons aussi que la manière dont Merleau-Ponty dévoile cette diversité déjà en œuvre dans l'activité perceptive nous permettra d'ajouter quelques suggestions au débat contemporain sur la perception initié par J. McDowell et peut-être excessivement limité à l'opposition entre conceptualistes et non-conceptualistes. Avant d'exposer les conséquences des réflexions de Merleau-Ponty, nous éclaircirons les raisons pour lesquelles les descriptions de la Phénoménologie de la perception, restent, en général, à un niveau préculturel.

\section{La perception : intentionnalité culturelle ou milieu universel?}

Reprenons rapidement la stratégie par laquelle Csordas s'approprie la notion d'expérience préobjective pour développer son paradigme anthropologique de l'incarnation. Csordas pense que cette notion permet de dévoiler le rôle constituant de l'activité subjective dans les relations avec le monde. Ainsi, si un anthropologue décrit l'expérience préobjective des individus, il peut comprendre, par des modalités intentionnelles diverses, comment ils organisent activement la vie sociale et culturelle. Csordas prend en considération une objection cruciale à sa conception: " on peut s'opposer au concept de préobjectif au sens où l'existence incarnée est en dehors ou antérieure à la culture ${ }^{2}$ ", ce qui le rendrait inutile au projet de saisir justement le développement de divers systèmes culturels. Contre cette objection, Csordas précise qu'en admettant le préobjectif «nous ne posons pas un préculturel, mais un préabstrait ${ }^{3}$ », c'est-à-dire un ensemble de relations qui ne sont pas encore explicitées et ordonnées conceptuellement, mais vécues de manière irréfléchie. Csordas affirme qu'on trouve cette conception du préobjectif déjà chez Merleau-Ponty. Selon lui, le phénoménologue français «a reconnu que la perception est toujours attachée à un monde culturel, si bien que le préobjectif n'implique nullement un "préculturel" ". L'anthropologue se réfère explicitement dans ce passage à la Phénoménologie de la perception, ce qui indique clairement selon lui que

1. T. Csordas, «Embodiment as a Paradigm for Anthropology». In Ethos, vol. 18, n 1, 1990, p. 40.

2. Ibid., p. 9.

3. Ibid., p. 10.

4. T. Csordas, «Somatic Modes of Attention». In Cultural Anthropology, vol. 8, n², 1993. p. 137. 
Merleau-Ponty considérait déjà dans ce livre le préobjectif comme culturel. Nous démontrerons ci-après que cette interprétation n'est pas correcte.

Tout d'abord, revenons à La structure du comportement, premier livre de Merleau-Ponty (publié en 1942), qui établit les principales lignes de recherche développées par le philosophe dans la Phénoménologie de la perception. Quelques extraits de La structure $d u$ comportement semblent confirmer la lecture de Csordas. Dans le troisième chapitre de ce livre, après avoir exposé l'apanage des ordres physique et vital, Merleau-Ponty cherche la spécificité de l'ordre humain et conclut que le travail humain "projette entre l'homme et les stimuli physicochimiques des "objets d'usage" [...] — le vêtement, la table, le jardin —, des "objets culturels" - le livre, l'instrument de musique, le langage - , qui constituent le milieu propre de l'homme et font émerger de nouveaux cycles de comportement ${ }^{5} »$. Ce milieu propre de l'être humain serait perçu immédiatement, c'est-à-dire qu'il ne serait pas composé d'une couche primordiale de stimuli objectifs auxquels on associerait postérieurement quelque sens culturel. La discrimination perceptive serait commandée par des impératifs propres à l'ordre humain, y compris les relations culturelles. Selon Merleau-Ponty, ce caractère immédiatement culturel de la perception devient évident si l'on étudie la conscience infantile, qui saisit éminemment, déjà dans les premières années de la vie, les gestes humains et les objets culturels. Par conséquent, la perception initiale n'assimile pas une nature objective comme celle étudiée par les sciences exactes; en effet, croit Merleau-Ponty, tous les stimuli sensibles donnent lieu, chez l'enfant, «à la représentation d'un drame humain» (SC, $182)^{6}$. Le philosophe français refuse que ce mode de conscience infantile soit un simple effet de la plus fréquente exposition des enfants à d'autres êtres humains et à des objets culturels. Il soutient que la conscience infantile est structurellement tournée vers le monde culturel. Au contraire, on ne saurait expliquer comment les êtres humains et le langage reçoivent si vite un privilège par rapport aux autres objets et bruits du champ sensible des enfants.

Jusqu'ici, l'argumentation de Merleau-Ponty lie intimement l'activité perceptive au monde culturel. Loin d'être expliquée comme une opération impartiale qui assimile passivement des stimuli objectifs, la perception est décrite comme une intentionnalité préconceptuelle qui nous rapproche de certains ensembles phénoménaux privilégiés, lesquels manifestent directement une signification culturelle. Toutefois, ce n'est pas le résultat ultime de Merleau-Ponty dans La structure du comportement. Le philosophe dévoile une ambiguité dans l'ordre humain: il est vrai que les sujets instituent des structures sociales ou culturelles où leur vie a lieu; cependant, la conscience humaine peut dépasser

5. M. Merleau-Ponty, La structure du comportement, Paris, PUF, 2002, p.175. Dorénavant citée comme SC dans le corps du texte.

6. Merleau-Ponty estime que les corps humains et les objets d'usage remplissent entièrement le champ de la perception infantile (cf. SC, 181). 
ces structures, les nier en tant que déterminants et s'affirmer comme milieu universel, c'est-à-dire, comme un champ où tous les objets de l'univers reçoivent leur signification (cf. SC, 191). Dans ce cas, on estime que la conscience est une espèce de foyer responsable par la manifestation de tout ce qui existe. Merleau-Ponty ne rejette pas l'idée que l'être humain est inséré dans des contextes sociaux et culturels. Mais il ajoute que, par rapport à cette consciencefoyer, «l'histoire même d'où elle sort n'est qu'un spectacle qu'elle se donne » (SC, 222), puisque tous les événements ou objets supposent la conscience pour qu'ils puissent se manifester et recevoir quelque sens. On doit noter qu'avec cette thèse Merleau-Ponty ne s'affilie pas à l'intellectualisme, vu que pour lui la conscience qui joue le rôle de milieu universel est la conscience perceptive (cf. SC, 227). C'est pour la perception que les phénomènes physiques, vitaux et même humains existent, et non pour le sujet cognitif. Selon MerleauPonty, ce dernier ne se rapporte qu'à des significations constituées subjectivement; par contre, la perception touche directement les choses du monde. En outre, du point de vue du sujet cognitif, le propre corps devient un objet soumis aux paramètres objectifs utilisés pour connaître des objets mondains. Déjà, du point de vue de la conscience perceptive, le propre corps est éprouvé comme son support indispensable, de sorte qu'elle doit être considérée comme incarnée (cf. SC, 224-5).

En dépit de ces différences entre la conscience perceptive et le sujet cognitif, la fonction que Merleau-Ponty confère à la première s'approche de celle exécutée par le second dans les philosophies intellectualistes: la conscience perceptive n'est plus conçue comme une capacité existentielle liée à quelques ensembles immédiatement significatifs, y compris les objets et les situations culturelles, mais comme champ transcendantal, dans lequel le monde réel s'organise en tant que phénomène significatif. Voilà la tendance qui prédomine dans la Phénoménologie de la perception, et qui semble l'éloigner d'une investigation anthropologique. D'après Merleau-Ponty, il n'est pas déterminant de présenter la conscience perceptive comme une intentionnalité essentiellement liée à des ensembles phénoménaux avec un sens symbolique-culturel, mais on doit plutôt «égaler la conscience à l'expérience entière » (SC, 240) et exami ner la perception comme un support universel par lequel la réalité se présente sous ses aspects les plus universels.

\section{L'organisation spontanée des phénomènes}

L'analyse de l'activité perceptive contenue dans la Phénoménologie de la perception est beaucoup plus détaillée que celle trouvée dans La structure $d u$ comportement. Cependant, l'idée générale de concevoir la perception comme un support universel de la réalité est maintenue dans le livre de 1945. On peut confirmer ce maintien par quelques affirmations de la préface de la Phénoménologie, où la perception est présentée non comme "un acte, une prise de 
position délibérée ${ }^{7}$ ", mais comme "le fond sur lequel tous les actes se détachent et [comme étant] présupposée par eux» (Php, V). La perception serait une "conscience originaire » $(\mathrm{Php}, \mathrm{X})$, antérieure au langage et à d'autres manifestations culturelles, une conscience autour de laquelle «s'organisent les actes de dénomination et d'expression" (id.) et où l'on a "accès à la vérité » (Php, XI). En outre, la conscience perceptive dispose d'un «savoir primordial du réel » $(i d$.$) , qui fonde la distinction entre réalité et rêve avant même que ce problème$ ait été explicité d'une manière conceptuelle. Ainsi, le champ préobjectif soutenu par la perception n'est pas une formation culturelle particulière, comme le début de l'exposé sur l'ordre humain dans La structure du comportement pourrait nous porter à le croire, mais il est plutôt une couche universelle responsable de l'insertion la plus générale des sujets humains dans le monde.

Comme nous l'avons déjà signalé, cette approche de la perception était déjà esquissée dans le premier livre de Merleau-Ponty. Le quatrième chapitre de La structure du comportement s'ouvre par un examen de l'expérience naïve, laquelle ne serait encore contaminée ni par des opinions du sens commun ni par des explications scientifiques. Il est remarquable qu'une telle expérience ne soit que celle du perspectivisme inhérent à la vision, selon le portrait qu'en fait la phénoménologie husserlienne. "Les "choses" dans l'expérience naïve sont évidentes comme êtres perspectifs [...]; je saisis dans un aspect perspectif dont je sais qu'il n'est qu'un de ses aspects possibles, la chose même qui le transcende " (SC, 202), nous raconte Merleau-Ponty. Cette description phénoménologique doit être au-dessus de toute particularité factuelle qui nuancerait ou complexifierait un tel perspectivisme. Selon le philosophe, quelle que soit la «difficulté qu'on trouve à penser la perception ainsi décrite, c'est à nous de nous en accommoder»(id.), puisque "c'est ainsi que nous percevons et que la conscience vit dans les choses » (id.). De plus, MerleauPonty est sûr d'exposer un caractère eidétique de la perception, indifférent à toute relativisation culturelle.

Dans la Phénoménologie de la perception, Merleau-Ponty reprend cette description des propriétés les plus générales de la perception. Le perspectivisme visuel est alors décrit comme structure figure sur fond: tout phénomène vu s'organise comme une figure, dont on ne voit que quelques aspects, et relevée d'un champ plus vaste. La structure figure sur fond est «la définition même du phénomène perceptif, ce sans quoi un phénomène ne peut être dit perception» $(\mathrm{Php}, 10)$. Cette définition du contenu perçu par la structure figure sur fond implique le refus de l'idée de sensation, comprise comme un stimulus ponctuel, instantané et objectivement délimitable. La sensation ainsi conçue ne décrit pas correctement le contenu perceptif, qui, selon Merleau-Ponty, est formé par relation entre figures et fonds.

7. M. Merleau-Ponty, Phénoménologie de la perception, Paris, Gallimard, 1997, p. V. Dorénavant citée dans le corps du texte comme Php. 
L'idée de sensation, encore d'après Merleau-Ponty, découle de la croyance en un monde objectif entièrement déterminé. Nous supposons que ce monde déterminé existe, et nous développons une théorie pour comprendre comment il nous est présenté: la sensation transposerait les qualités objectives du monde jusqu'à la conscience. Or, pour Merleau-Ponty, la thèse d'une reproduction dans la conscience perceptive de caractères d'un monde conçu objectivement (avec des propriétés mensurables, clairement distinguables, etc.) n'est pas correcte. Ceux qui soutiennent cette thèse ignorent l'organisation propre de l'expérience perceptive. De l'avis de Merleau-Ponty, cette organisation dévoile au moins deux caractères qui dépassent l'idée de sensation. Le premier est l'indétermination perceptive, selon laquelle les ensembles phénoménaux ne se réduisent pas à des propriétés exactes. Les lignes dans l'illusion de Müller-Lyer, qui ne peuvent être dites ni égales ni inégales, illustrent ce caractère (cf. Php, 12). Et non seulement les illusions optiques sont indéterminées, mais, en général, tout ensemble phénoménal renferme quelque degré d'indétermination, vu que sa signification se constitue non pas par des mesures figées mais plutôt par d'innombrables relations entretenues parmi les éléments perçus. Le deuxième caractère signalé par Merleau-Ponty est l'aspect lacunaire de la perception. D'après lui, la manifestation du sens des phénomènes ne dépend pas d'une correspondance point par point à une couche de sensations objectives. Le phénoménologue propose quelques exemples de cas où le phénomène perçu ne se restreint pas aux données sensibles: «la force du son sous certaines conditions lui fait perdre de la hauteur, l'adjonction de lignes auxiliaires rend inégales deux figures objectivement égales» (Php, 14). Dans ces cas, ce qu'on perçoit ne se réduit pas aux informations objectives contenues dans les données sensibles, mais suit les paramètres d'organisation propres du champ phénoménal.

Ces deux caractères déterminent la signification préobjective des phénomènes perçus, que Merleau-Ponty essaie d'exposer tout au long de Phénoménologie de la perception. Or il nous faut remarquer que, selon l'analyse développée dans ce livre, cette signification est conçue comme antérieure à toute qualification culturelle. Cela devient clair si l'on reprend la méthode suggérée par Merleau-Ponty pour étudier la signification perceptive. Le phénoménologue préconise d'excéder la "perception empirique ou seconde, celle que nous exerçons à chaque instant» (Php, 53-4), puisqu'elle masque l'organisation originaire du champ phénoménal. Cette perception empirique «est toute pleine d'acquisitions anciennes et se joue pour ainsi dire à la surface de l'être » (Php, 54); bref, elle est chargée de significations culturelles et présente les choses selon leur fonctionnalité dans les contextes vécus. Cette perception cultivée doit être mise entre parenthèses en faveur d'une contemplation désintéressée qui saisit « un objet avec le seul souci de le voir exister et déployer [...] ses richesses (id.). Merleau-Ponty formule au moins un exemple de cet effort contemplatif où la productivité inhérente à la perception, en-deçà des significations sédimentées sur les phénomènes, se manifeste: "pour que je reconnaisse 
l'arbre comme un arbre, il faut que, par-dessous cette signification acquise, l'arrangement momentané du spectacle sensible recommence, comme au premier jour du monde végétal, à dessiner l'idée individuelle de cet arbre » (id.). Ce retour au commencement presque mythique de l'activité perceptive, où les relations du champ perceptif seraient libres de toute signification acquise et pourraient montrer leur concaténation naturelle, exclut toute densité culturelle, afin de rendre évidents des processus d'organisation sensible de caractère universel.

Si l'on s'éloigne des perceptions secondes et si l'on suit le recommencement perpétuel de la structuration du monde perçu, que trouve-t-on? Selon Merleau-Ponty, on trouve un processus général de distribution des qualités perçues (les couleurs, les formes, les sons, etc.) par lequel tout phénomène est organisé ${ }^{8}$. Cette distribution ne suit aucune détermination objective, mais des lignes de force immanentes à la situation perçue 9 . Il faut noter que cette distribution des qualités perçues selon l'ensemble phénoménal impose un ordre, malgré son caractère préobjectif, vraiment inflexible. Selon Merleau-Ponty, les couleurs, les formes, le son, l'odeur des objets perçus sont intimement liés, de sorte que la perception engendre des phénomènes réunis dans un réseau expressif rigide, où chaque aspect renvoie aux autres d'une manière exhaustive, laquelle exclut ce qui ne s'harmonise pas au résultat visé. Pour le philosophe français, « une chose n'aurait pas cette couleur si elle n'avait aussi cette forme, ces propriétés tactiles, cette sonorité, cette odeur » (Php, 368), si bien que les données des différents sens s'entrecroisent d'une manière nécessaire dans la présentation du monde perçu ${ }^{11}$.

Comme on le voit, dans la Phénoménologie de la perception, l'activité perceptive organise les données sensibles selon la configuration globale des phénomènes en question, et les résultats perçus constituent un spectacle

8. Par exemple, "soit un mur blanc dans l'ombre et un papier gris à la lumière, on ne peut pas dire que le mur reste blanc et le papier gris " (Php, 351). Cet exemple illustre bien le type d'analyse effectuée par Merleau-Ponty dans la Phénoménologie de la perception. Il s'agit d'éclaircir comment les composants universels des phénomènes perçus s'arrangent selon les restrictions inhérentes aux structures perceptives.

9. «On n'a pas dans le champ originaire une mosaïque de qualités, mais une configuration totale qui distribue les valeurs fonctionnelles selon l'exigence de l'ensemble » (Php, 279).

10. "Notre perception tout entière est animée d'une logique qui assigne à chaque objet toutes ses déterminations en fonction de celles des autres et qui "barre" comme irréelle toute donnée aberrante » (Php, 361).

11. Rappelons que cette unité de l'objet perçu est toujours présumable, vu que la synthèse perceptive est essentiellement temporelle: " chaque aspect de la chose qui tombe sous notre perception n'est encore qu'une invitation à percevoir au-delà et qu'un arrêt momentané dans le processus perceptif» (Php, 269-270). En effet, la concaténation rigide des aspects perçus indique que la description de la signification perceptive, qui est indéterminée (comme nous l'avons vu plus haut), n'implique pas que le champ phénoménal soit une région floue, où n'importe quelle relation est possible. Il y a indétermination par rapport à des catégories objectives, mais non par rapport aux résultats perçus dans le champ phénoménal. 
rigidement construit, où chaque élément sensible se rapporte à tous les autres d'une manière définitive. Cette thèse exige quelques éclaircissements. On peut se demander si le spectacle perçu s'impose comme étant le même à toutes les personnes. Merleau-Ponty ne répond pas clairement à cette question. Mais il formule quelques exemples de l'expressivité des données sensibles qui nous permettent de réfléchir à ce propos: «on voit la rigidité et la fragilité du verre et, quand il se brise avec un son cristallin, ce son est porté par le verre visible. On voit l'élasticité de l'acier, la ductilité de l'acier rougi, la dureté de la lame dans un rabot, la mollesse des copeaux» (Php, 265). On se demande si cette reconnaissance immédiate de la richesse expressive du verre ou de l'acier ne suppose pas un long contact avec eux. Peut-être que des peuples qui ne connaissent pas la métallurgie ou la vitrerie, s'ils étaient exposés à ces matériaux, ne percevraient pas les détails expressifs tels que Merleau-Ponty les décrit. Dans ce cas, les résultats perçus dépendraient d'un apprentissage culturel et non seulement d'une organisation spontanée des données sensibles. Il est vrai que Merleau-Ponty mentionne que la couche perceptive originaire n'est pas anhistorique. Il y aurait une «tradition perceptive» $(\mathrm{Php}, 275)$ sur laquelle la vie personnelle s'érige. Mais il ne précise pas si cette tradition est universelle ou s'il y en a plusieurs, ni si elle peut changer ou si elle forme une couche statique. En général, si l'on suit les descriptions de la Phénoménologie de la perception, il semble que le champ préobjectif de la perception (lequel ne se confond pas avec la perception seconde et chargée de significations pratiques) soit composé par des processus universels, qui se développent à l'écart de la diversité culturelle.

\section{Le corps et la fondation de la culture}

Nous avons essayé de montrer jusqu'ici que, selon la Phénoménologie de la perception, l'activité perceptive n'est pas attachée à un milieu culturel. En effet, dans ce livre, Merleau-Ponty soutient que les processus d'organisation perceptive font partie «des montages naturels du sujet psycho-physique»(Php, 59), c'est-à-dire, d'un niveau antérieur à l'apprentissage culturel et potentiellement partagé par tous les hommes. Le phénoménologue reconnaît «que les structures perceptives ne s'imposent pas toujours» (Php, 503), et que des relations symbolico-culturelles influent parfois sur la ségrégation des phénomènes, comme La structure du comportement l'avait déjà exposé. Toutefois, cette petite remarque n'éclaircit en rien comment les composantes culturelles aideraient à informer la vie perceptive, laquelle est présentée, au moins dans presque toute la Phénoménologie de la perception, comme préculturelle.

Malgré cette hantise du préculturel, il serait extrêmement simplificateur de conclure que la Phénoménologie de la perception ignore complètement le rôle de la diversité culturelle dans la constitution de l'expérience. En effet, pour lui attribuer ce rôle, il faut suivre le thème du propre corps, c'est-à-dire, non pas le corps objectif (fragment de matière recherché par les sciences biologiques), mais le corps vif, toujours en contact avec quelque situation concrète. 
On doit remarquer que, selon la Phénoménologie, la perception est une activité incarnée (comme La structure du comportement le signalait déjà), de sorte que ses caractéristiques se fondent dans la vie corporelle ${ }^{12}$. Il faut noter cependant que l'activité perceptive est un type très spécial d'habileté corporelle, vu qu'une telle activité engendre un champ de phénomènes organisés de manière rigide, qui ne tolère pas de variations (cf. Php, 390). Cette rigidité implique que le spectacle perçu est un tissu constitué par des relations indépendantes des décisions personnelles ou des variables culturelles. Déjà, dans d'autres activités (par exemple, l'acquisition d'une habitude), le corps se sert de tous ses subsystèmes organiques pour exprimer diverses manières de s'insérer dans le monde. Merleau-Ponty souligne que "l'usage qu'un homme fera de son corps est transcendant à l'égard de ce corps comme être simplement biologique» (Php, 220). Il est vrai, par exemple, que tous les êtres humains doivent satisfaire les impératifs biologiques du sommeil et de la nutrition; mais il est vrai aussi qu'ils ne le font pas de la même manière. Par conséquent, les nécessités biologiques universelles n'impliquent pas la formation de procédés uniques mais de plusieurs manières de vivre le corps.

Il est vrai que Merleau-Ponty signale quelque caractère nécessaire dans l'usage du corps; mais ce caractère n'est pas du côté des conséquences du processus intentionnel mais de celui du processus lui-même. Ainsi, tous les aspects anatomiques et physiologiques du corps collaborent nécessairement pour établir les différentes habitudes par lesquelles on s'insère dans le monde ${ }^{13}$. Mais cela n'implique pas qu'il y ait une seule façon de s'insérer corporellement dans le monde, comme il semblait y avoir une seule organisation possible du spectacle perçu, imperméable aux particularités culturelles et socio-historiques. Merleau-Ponty le reconnaît: «l'équipement psychophysiologique laisse ouvertes quantités de possibilités et il n’y a pas plus ici que dans le domaine des instincts une nature humaine donnée une fois pour toutes» (Php, 220). Il cite comme exemple les différences dans l'expression des émotions chez les Occidentaux et les Japonais, et souligne que «la différence des mimiques recouvre une différence des émotions elles-mêmes» (id.). Ainsi, loin d'être vécues d'une manière unique, les émotions, même les plus incrustées dans l'agir humain, «sont inventées comme les mots» (id.) et se sédimentent de sorte à cristalliser plusieurs contextes culturels.

12. «Le sentir [...] investit la qualité d'une valeur vitale, la saisit d'abord dans sa signification pour nous, pour cette masse pesante qui est notre corps, et de là vient qu'il comporte toujours une référence au corps» (Php, 64).

13 «Si [...] on définit l'homme par son expérience, c'est-à-dire par sa manière propre de mettre en forme le monde, et si l'on réintègre les “organes" à ce tout fonctionnel dans lequel ils sont découpés, un homme sans mains ou sans système sexuel est aussi inconcevable qu'un homme sans pensée » (Php, 198). Dans la même page, Merleau-Ponty affirme: «il est impossible de distinguer dans l'être total de l'homme une organisation corporelle, que l'on traiterait comme un fait contingent, et d'autres prédicats qui lui appartiendraient avec nécessité » (id.) 
Comme on l'observe, le champ de l'expérience préobjective corporelle implique la relativité culturelle, une thèse qui semble nourrir une oscillation interne à la pensée de Merleau-Ponty. D'un côté, le retour au champ exclusivement perceptif révèle des ensembles phénoménaux soudés par une expressivité rigide, étrangère à la diversité culturelle. D'un autre côté, le retour à l'expérience corporelle ne dévoile pas de résultats uniques, mais différents usages du corps. Néanmoins, dans certains passages, Merleau-Ponty semble assujettir la diversité instaurée par le corps à l'universalité véhiculée par la perception. Le phénoménologue soutient que le corps lui-même projette comme milieu non seulement une spatialité anthropologique liée aux manières particulières de vivre les situations confrontées, mais premièrement un fond commun et objectivable, qui se manifeste clairement dans l'expérience normale: «je ne vis pas entièrement dans les espaces anthropologiques, je suis toujours attaché par mes racines à un espace naturel et inhumain» (Php, 339). L'entrée du corps dans le monde culturel suppose, bien que d'une manière implicite, son insertion "d'abord dans un monde naturel qui transparaît toujours sous l'autre» (id.). En effet, l'existence humaine relève des " "mondes" sur le fond d'un unique monde naturel» (Php, 340), et ce monde premier, en-deçà de la relativité culturelle, serait justement celui procuré par l'activité perceptive. Ainsi, non seulement Merleau-Ponty conçoit la perception comme un noyau imperméable à la culture, mais il y soumet toute activité corporelle, de façon à fonder les résultats multiples de l'expressivité du corps sur l'universalité silencieuse du champ perceptif. De cette manière, l'institution de divers milieux culturels dépend d'un sol universel préalable, c'est-à-dire de l'élaboration d'un champ où la nature phénoménale se manifeste selon une expressivité infranchissable, laquelle présente le champ préobjectif (au moins à son niveau le plus originaire) comme préculturel. Dans ce sens, même si l'on se réfère aux descriptions merleaupontyennes de l'usage du corps, il n'est pas possible d'interpréter le préobjectif, tel que présenté par la Phénoménologie de la perception, comme culturellement informé, car le phénoménologue soumet ces descriptions au primat de l'activité perceptive comme organisation préculturelle d'un monde phénoménal universellement partagé.

\section{La période intermédiaire de Merleau-Ponty}

Selon les thèses de la Phénoménologie de la perception, il n'est pas correct d'affirmer que le champ de phénomènes préobjectifs est déjà culturel, comme le voudrait Csordas. Cependant, l'interprétation que l'anthropologue présente de Merleau-Ponty peut être confirmée si l'on considère d'autres œuvres du philosophe français. Il est remarquable que dans certains écrits des années cinquante, Merleau-Ponty essaie de concevoir la perception comme immédiatement culturelle. Le phénoménologue considère alors acceptable la thèse que 
«notre culture peut $[\ldots]$ informer notre perception du visible ${ }^{14} »$, ou que la vie perceptive "projette dans le monde la signature d'une civilisation ${ }^{15}{ }$. Comment Merleau-Ponty arrive-t-il à ces affirmations?

Il nous semble que le philosophe français atteint un tel résultat surtout par une approximation entre parole et perception. Dès La structure du comportement, Merleau-Ponty décrit la perception comme une activité qui contracte des données partielles et en présente une chose complète, dont l'explicitation de la totalité de ses aspects exigerait un temps infini. Dans ce cas, la perception se cache elle-même comme un événement partiel en faveur de choses pleines. Pareillement, le langage tel que décrit dans les années cinquante «se [fait] oublier dans la mesure où il réussit à exprimer ${ }^{16}$ ", c'est-à-dire que les actes expressifs qui constituent nos concepts se dissipent en faveur d'expressions accomplies, et on a alors l'impression que ces concepts sont des entités autonomes et indépendantes de l'activité subjective. Ainsi, Merleau-Ponty considère que perception et langage excèdent leurs données initiales, vu que tant une chose perçue qu'un signifié visé sont toujours affirmés par les actes perceptifs ou phoniques, mais ces actes, en effet, ne saisissent que des aspects partiels des phénomènes ou des expressions linguistiques ${ }^{17}$. Toutefois, ce point ne suffit pas encore pour approcher vraiment perception et langage, comme on le verra plus loin.

Merleau-Ponty soutenait, nous l'avons vu, que les résultats de l'activité perceptive sont organisés d'une manière rigide, qui ne comporte pas de variations. Ainsi, la transcendance des stimuli partiels vers l'objet perçu présenterait toujours des qualités perceptives réunies selon une logique universelle. Cependant, cela n'arrive pas avec la parole. Même dans la Phénoménologie de la perception, les significations visées par les actes linguistiques ne sont pas définitives, c'est-à-dire qu'elles peuvent être modifiées, développées, interprétées, etc. Il n'y aurait pas de significations rigides dans le sens où leur contenu ne comporterait pas de variations. Les significations linguistiques sont considérées par Merleau-Ponty comme des ressources partielles qui sédimentent une base pour des actes linguistiques qui créent de nouveaux sens. Ceuxci surgissent par de nouveaux usages des significations sédimentées, de sorte que les possibilités expressives contenues en elles ne s'épuisent en aucune de leurs formulations (cf. Php, «Le corps comme expression et la parole»).

Dans la Phénoménologie de la perception, cette propriété de sédimentation d'une base de résultats partiels et modifiables était attribuée seule à la

14. M. Merleau-Ponty, "Le langage indirect et les voix du silence». In Signes, Paris, Gallimard, 1960, p. 61. Dorénavant mentionné comme $S$ dans le corps du texte. Cet article a été originellement publié dans Les Temps modernes en 1952.

15. M. Merleau-Ponty, La prose du monde, Paris, Gallimard, col. Tel, 1999, p. 97. Dorénavant mentionné comme PM dans le corps du texte. Ce texte a été écrit entre 1949 et 1952.

16. M. Merleau-Ponty, La prose du monde. Paris, Gallimard, col. Tel, 1999, p. 15.

17. Cf. M. Merleau-Ponty, "Sur la phénoménologie du langage». In Signes, Paris, Gallimard, 1960, p. 114. Dorénavant mentionné comme $S$ dans le corps du texte. 
parole (cf. Php, 221). Or, pour vraiment rapprocher le mode de fonctionnement de la perception et de la parole, Merleau-Ponty doit attribuer à la première cette cristallisation de résultats non définitifs. En effet, dans les textes des années cinquante, le philosophe français semble admettre que la perception saisit les phénomènes d'une manière analogue à la parole. Il ne s'agit pas d'affirmer que le processus de viser un objet moyennant des données partielles présente ce même objet selon une ordonnance qui ne tolère pas de variations. En effet, un tel processus sédimente quelques approches privilégiées sur tel objet, de sorte que les résultats perçus ne constituent pas un monde phénoménal unique, comme le décrivait la Phénoménologie de la perception, mais cristallisent une manière partielle, informée culturellement, d'avoir accès au champ perçu.

Dans la Phénoménologie de la perception, Merleau-Ponty reconnaissait que les choses perçues se manifestent sur des données toujours partielles (cf. Php, 84). Cependant, dans ce livre, l'inachèvement de l'appréhension perceptive n'impliquait pas la possibilité d'organisations divergentes du champ perceptif. Déjà, dans ses textes intermédiaires, Merleau-Ponty admet que le fait que le champ perçu s'érige toujours sur des données partielles pour présenter les phénomènes indique que la perception n'arrive pas à exposer les relations expressives parmi les données d'un mode définitif. Au contraire, l'activité perceptive, selon ces textes, cristallise des étalons particuliers d'assimilation des données, c'est-à-dire qu'elle favorise des habitudes perceptives qui nourrissent des discriminations plus récurrentes de quelques traits perceptifs. De cette manière, Merleau-Ponty conclut de la thèse de l'inachèvement phénoménal que les résultats perceptifs ne sont pas toujours vécus d'une manière universelle. En réalité, ces résultats doivent être conçus comme des sédimentations d'une appréhension culturelle déterminée du monde perçu. Les étalons sensibles sédimentés n'épuisent pas toutes les possibilités expressives contenues dans le spectacle phénoménal, qui, pour Merleau-Ponty, n'exige ni ne refuse aucune des déterminations historique-culturelles cristallisées, mais les contient toutes comme des possibilités latentes de configurations phénoménales ${ }^{18}$.

Le thème des habitudes perceptives est exploré par Merleau-Ponty dans l'article «Le langage indirect et les voix du silence », où le phénoménologue conteste l'interprétation classique de la peinture réaliste selon laquelle cette peinture, moyennant quelques ressources techniques (telles que la perspective planimétrique), représente la réalité d'une telle manière que ses résultats seraient reconnus universellement par tous les êtres humains. Cette interprétation, qui pourrait bien être soutenue par la Phénoménologie de la perception, prend le

18. De cette manière, l'idée de tradition perceptive mentionnée dans la Phénoménologie de la perception (cf. Php, 275) peut effectivement gagner du sens. Ce livre présentait l'organisation perceptive comme un processus qui procure des résultats universellement partageables d'une manière immédiate; par conséquent, on ne voyait pas comment la notion de tradition, qui paraît inclure des procédés partiels et changeables, pourrait être appliquée à la perception. 
système perceptif «comme un moyen naturel et donné de communication entre les hommes » $(S, 60)$, qui les lierait à un monde unique; elle suppose encore que "les "données des sens" à travers les siècles n'aient jamais varié » $(S, 61)$, c'est-à-dire qu'il y ait un contenu perceptif qui s'impose nécessairement à toutes les cultures à travers les âges. Merleau-Ponty rejette cette thèse; pour lui « la perception des classiques relevait de leur culture» (id.), et leur peinture, loin de traduire le monde réel manifesté de manière inexorable par les sens, exprime un monde dominé par la connaissance humaine, c'est-à-dire exprime une expectative culturelle particulière en vigueur à l'époque du rationalisme classique.

Il s'ensuit de ces affirmations que le monde perçu n'est pas un tissu phénoménal composé de significations universellement appréhendées, mais un champ polymorphe dont quelques configurations sont privilégiées historiquement. Ainsi, les objets perçus incluent une latence de signification qui ne s'épuise en aucune de leurs manifestations phénoménales. Mais ces manifestations, à leur tour, sont toujours vécues selon quelques déterminations culturelles, de sorte que les phénomènes perçus ne sont plus décrits par Merleau-Ponty dans les années cinquante comme une couche préculturelle, mais comme une expression de déterminations historiques. Ainsi, au moins dans ses textes intermédiaires, le philosophe présente une analyse de l'activité perceptive qui confirme finalement l'interprétation de Csordas, selon laquelle le champ préobjectif est déjà culturel. Cette interprétation de Merleau-Ponty, qui sous-tend le paradigme anthropologique de l'incarnation, peut aussi, croyons-nous, renouveler quelques aspects de la discussion contemporaine sur la perception, comme on le verra ci-dessous.

\section{Note pour le débat contemporain sur la perception}

Au moins dès la publication de l'œuvre décisive de J. McDowell, Mind and World, plusieurs auteurs discutent la relation entre le contenu perceptif et les croyances rationnelles, et, en général, essaient d'éclaircir jusqu'à quel point les données perceptives peuvent servir à justifier des propositions théoriques. McDowell soutient que les données sensibles ne sont pas reçues comme un amas désorganisé. Il y aurait un sens dans les phénomènes perçus qui serait délimité, en fin de compte, par la rationalité humaine. Selon McDowell, les «capacités conceptuelles [...] peuvent être opératives non seulement dans les jugements [...] mais déjà dans les rapports qui sont constitués par les chocs du monde avec les capacités réceptives d'un sujet convenable ${ }^{19}$ ". De ce point de vue, il y aurait une homogénéité entre sensibilité et entendement, de sorte que l'activité perceptive actualiserait les capacités conceptuelles subjectives. Aussi faudrait-il, pour avoir l'expérience d'une chose ou d'une situation, posséder les concepts qui permettent de distinguer correctement son apparition. 
Contre ces thèses, quelques auteurs soutiennent que la donnée perceptive ne se réduit pas à l'actualisation des capacités conceptuelles. W. Wright illustre bien cette position. Pour lui, «il semble y avoir beaucoup de cas où il est approprié d'attribuer à un sujet des expériences avec des contenus auxquels ne correspond aucun concept ${ }^{20} »$. De ce point de vue, le contenu perceptif utilisé pour justifier des croyances rationnelles pourrait s'organiser indépendamment de l'existence de concepts correspondants, moyennant, par exemple, les règles générales de la prégnance gestaltiste. Selon ce point de vue, dans n'importe quelle expérience perceptive, il y aurait une infinité d'aspects raffinés (des tonalités, des formes, des bruits, des odeurs), dont les propriétés et relations, quoiqu'elles ne soient pas appréhendées conceptuellement, figurent d'une manière organisée dans le champ perceptif. Nous n'explorerons pas en détail les arguments en faveur de ces deux positions. Nous ne voulons que marquer l'originalité de Merleau-Ponty par rapport à ces positions générales.

Cette opposition entre conceptualistes et non-conceptualistes est bouleversée si l'on met en jeu les positions de Merleau-Ponty, telles que nous les avons exposées dans notre dernière section. Le phénoménologue ne se demande pas si les capacités conceptuelles et les données sensibles se recoupent, mais si un tel recoupement a lieu entre les données sensibles et un contexte historicoculturel. Cette nouvelle dimension du problème suggère une solution médiane à l'opposition entre conceptualistes et non-conceptualistes, comme on le verra. Pour rendre cette solution plus visible, il serait envisageable d'éclaircir la nouveauté des thèses de Merleau-Ponty par rapport à celles de Heidegger.

En général, les thèses de Merleau-Ponty sur la perception, dans les années cinquante, se rapprochent de celles de Heidegger, qui rejette expressément la primauté de l'activité perceptive (conçue comme purement sensorielle) quant à l'insertion humaine dans le monde. Pour le philosophe allemand, cette insertion a lieu selon la compréhension préconceptuelle de l'Être, c'est-à-dire comme une relation historico-culturelle qui délimite le sens de tout ce dont on peut avoir l'expérience. Ainsi, le sens de ce qui est perçu est ordonné selon les relations culturelles d'une époque historique. Heidegger donne l'exemple suivant: "Ce que l'oreille perçoit et la manière dont elle perçoit se trouvent déjà qualifiés et déterminés par ce que nous entendons ${ }^{21}$.» En d'autres termes, l'acte de percevoir ne présente pas par lui-même un spectacle significatif; il y aurait un sens historico-culturel préalable (une saisie préconceptuelle du monde et des étants) qui informe et dirige la perception. Il nous semble que Merleau-Ponty, dans les textes des années cinquante, pourrait bien corroborer cette thèse heideggérienne.

20. W. Wright, «McDowell, Demonstrative Concepts, and Non Conceptual Representational Content ». In Disputatio, n 14, 2003, p. 41.

21. M. Heidegger, Le principe de raison, Paris, Gallimard, 1983, p. 124. 
Même s'il y a une proximité avec Heidegger, on doit souligner la singularité de la position de Merleau-Ponty. Le philosophe français admet que dans la perception se cristallisent des étalons historico-culturels déterminés, thèse avec laquelle Heidegger serait probablement d'accord; mais Merleau-Ponty défend aussi que l'activité perceptive n'exige aucun étalon culturel en particulier ni ne s'épuise en aucun d'eux. Cela veut dire que la perception ne se réduit pas du tout à actualiser un tissu de significations historiques; on décrirait mieux l'activité perceptive en affirmant qu'elle soutient un champ de données sensibles dont plusieurs relations latentes peuvent être différemment explorées par différents systèmes culturels, un champ qui les tolère toutes sans s'engager en définitive avec aucun d'eux. Par conséquent, la position de Merleau-Ponty conserve la possibilité que la perception saisisse des configurations phénoménales qui non seulement vérifient les conceptions tacites d'un contexte historico-culturel, mais aussi les ébranlent. D'un point de vue merleau-pontyen, la perception peut saisir des configurations difficilement réductibles à des actualisations d'une compréhension préalable de l'Être, configurations qui, au contraire, exigent des altérations dans cette compréhension. Ainsi, Merleau-Ponty ne soumet pas complètement la perception aux étalons historico-culturels et nous permet de considérer entre eux des relations de détermination réciproque. Cette conséquence découle de la thèse de la latence polymorphe du champ phénoménal, laquelle ne se trouve pas clairement chez Heidegger, mais qui sans doute est défendue par Merleau-Ponty.

Comme on le voit, il y a au moins deux points remarquables dans la position de Merleau-Ponty sur la perception dans les années cinquante. D’un côté, il reconnaît que, plus que des arrangements universels de couleurs ou de sons, la perception engendre quelques phénomènes récurrents, dont la discrimination découle d'habitudes et d'expectatives culturellement informées. D'un autre côté, il soutient que la perception ne s'épuise pas dans l'actualisation de ces habitudes ou expectatives mais peut présenter des phénomènes qui excèdent le contexte historico-culturel en question. Un exemple rendra plus clair ce double aspect.

Pensons aux peuples amazoniens qui n'ont pas de contact avec la civilisation occidentale ${ }^{22}$. Ces peuples ont exercé leurs capacités perceptives dans un contexte très spécifique (la forêt amazonienne) et probablement développé des habiletés discriminatoires très raffinées dans ce contexte. Ils peuvent distinguer, par exemple, le chant d'un oiseau et le cri d'un sagouin, alors que tous les deux paraîtraient très semblables à des personnes non accoutumées à ces animaux. Un habitant d'une grande métropole internationale, qui n'a jamais vécu dans une forêt ni étudié la zoologie, ne distinguerait probablement pas

22. Récemment, en mai 2007, au Brésil, un groupe d'indigènes Metyktire qui s'est isolé pendant plus de cinquante ans a repris le contact avec la civilisation occidentale. Certainement, les plus jeunes du groupe ne connaissent pas les éléments les plus communs des villes contemporaines, tels que l'électricité, la circulation, etc. 
les gazouillements des sifflements de plusieurs bêtes de la forêt amazonienne. Ce citoyen métropolitain, mis en situation, ne serait pas non plus capable de distinguer l'oiseau « urutal » d'un tronc d'arbre, non seulement parce que cet oiseau se camoufle presque parfaitement sur les troncs d'arbres, mais aussi parce que notre citoyen, qui ne connaît, par hypothèse, rien de l'ornithologie brésilienne, ne peut même pas concevoir qu'un oiseau puisse se confondre avec un tronc d'arbre, c'est-à-dire qu'il n'ait pas la disposition culturelle à extraire ce phénomène des données sensibles reçues ${ }^{23}$.

Il est clair que la possibilité de saisir significativement plusieurs phénomènes de la forêt amazonienne suppose un long apprentissage. Pareillement, si l'on transférait dans une grande ville un individu d'un de ces peuples amazoniens qui n'ont pas de contact avec la civilisation occidentale, il ne distinguerait probablement pas le bruit d'une voiture de celui d'un camion. La masse de sons d'une autoroute embouteillée ne serait pas assimilée, de telle sorte qu'elle susciterait des associations entre les types de véhicules qui y roulent et le type de son que l'on y entend. Cependant, tant le citoyen métropolitain dans la forêt que l'Amazonien dans une grande ville entendraient certainement des bruits ou verraient des figures du milieu où ils sont insérés, même s'ils ne les comprenaient pas immédiatement dans toute leur complexité.

Cet exemple du changement de contexte entre l'Amazonien et le citoyen métropolitain éclaircit, en premier lieu, le fait que le même ensemble de données sensibles favorise différentes discriminations phénoménales (par exemple, dans un certain paysage amazonien, l'Amazonien verrait un urutal parmi quelques troncs, mais le citoyen métropolitain ne verrait que des arbres). Ainsi, les articulations discriminées dans le champ phénoménal ne sont pas absolument rigides, comme le défendait la Phénoménologie de la perception; il est possible que quelques aspects du champ soient plus soulignés que d'autres. Ce qu'on peut percevoir dans un paysage n'est pas saisi simplement parce qu'il $\mathrm{y}$ a des règles universelles de prégnance gestaltiste. Il y a différents paramètres de discrimination, qui tiennent à la sédimentation d'habitudes et d'intérêts informés culturellement.

En second lieu, notre exemple montre que la perception peut présenter des phénomènes qui excèdent les paramètres culturels par lesquels les données sensibles sont normalement saisies. Notre citoyen imaginaire dans la forêt amazonienne pourrait par hasard voir un urutal en mouvement et l'identifier à un type d'oiseau qui se confond avec les troncs. À son tour, notre natif amazonien transporté dans la métropole serait bouleversé d'une manière beaucoup plus forte (puisque le citoyen, sans rien connaître de l'ornithologie brésilienne, saurait au moins qu'il y a des forêts et d'étranges animaux dans

23. Et même si le citoyen savait (théoriquement) qu'il y a des oiseaux qui se camouflent sur un tronc d'arbre, cela n'implique pas qu'il serait en mesure d'identifier (pratiquement) un oiseau lorsqu'il se camoufle sur le tronc d'arbre. 
le monde); il serait envahi par une foule de bruits et de formes qui, probablement, surpasseraient tout ce qu'il peut concevoir du monde. Il faut noter que, même si ce natif ne pouvait pas saisir intelligiblement tous les phénomènes perçus dans le nouveau milieu, ces phénomènes s'imposeraient, y compris à un degré très bas de raffinement et d'ordonnancement.

Ces considérations nous amènent à revoir l'opposition générale entre conceptualistes et non-conceptualistes dans le débat contemporain sur la perception. D'un point de vue merleau-pontyen, les conceptualistes soulignent correctement le fait qu'en général les données sensibles ne sont pas un amas désordonné, mais des ensembles présentés selon une configuration immédiatement significative. Mais, encore selon le point de vue de Merleau-Ponty, les non-conceptualistes remarquent avec justesse que ce sens n'est pas seulement conceptuel. Par conséquent, une solution médiane s'impose: il y aurait en effet une ordonnance immédiate des données sensibles, mais selon quelques lignes de force civilisationnelles, composées de coutumes, d'habiletés pratiques, de particularités linguistiques, de paradigmes existentiels, d'économie de pulsions, c'està-dire, de différents éléments de la culture qui sédimentent quelques habitudes perceptives. Ces habitudes, à leur tour, favorisent la discrimination d'une gamme déterminée de phénomènes parmi toutes les possibilités du champ perceptif.

Pour signaler le caractère médian de cette solution, on doit noter, tout d'abord, que les étalons perceptifs cristallisés par ces lignes de force culturelles ne se limitent pas à actualiser des capacités conceptuelles mais expriment tout un milieu existentiel, dont le sens est plus vaste que celui formulable conceptuellement. Merleau-Ponty a défendu ce point vigoureusement dans la Phénoménologie de la perception. Selon lui, pour comprendre le champ phénoménal, il faut « reculer les limites de ce qui a sens pour nous et replacer la zone étroite du sens thématique dans celle du sens non thématique qui l'embrasse " (Php, 318). De ce point de vue, le contenu conceptuel ne serait que le sens thématique, c'est-à-dire l'explicitation prédicative de l'expérience. Il y aurait, au-delà de ce contenu, une zone large de sens qui embrasse des relations historico-culturelles difficiles à traduire conceptuellement, mais selon lesquelles le champ préobjectif est aussi organisé. Prenons encore l'exemple de l'Amazonien, mais en le considérant en l'espèce inséré dans son contexte naturel, la forêt. Il est probable qu'il peut distinguer divers types de plantes par les seules nuances de vert, ou même savoir par la couleur de ses feuilles si une plante est malade ou non. Mais voici le point où les non-conceptualistes ont raison par rapport aux conceptualistes: l'Amazonien ne possède probablement pas de concepts correspondants à ces discriminations raffinées. Il n'est pas suffisant de recourir à des concepts démonstratifs tels que "ce vert-là » ou « un vert comme celui-ci », pour soutenir que même le contenu raffiné de l'expérience dépend d'une armature conceptuelle. Comme Wright l'argumente, à 
notre avis d'une manière convaincante, ces concepts démonstratifs, loin d'organiser le champ perceptif, dépendent de la manifestation actuelle du contenu raffiné en question (dans ce cas, différentes tonalités de vert) pour qu'ils puissent être utilisés ${ }^{24}$. On peut, par exemple, désigner «ce vert-là » comme une tonalité appelée «vert 25 ». Mais on ne peut reconnaître d'autres cas de « vert 25 » sans percevoir, de manière concomitante, un étalon de mesure « vert 25 ». Les références démonstratives au "vert 25 » ne permettent pas de reconnaître d'autres échantillons de la même tonalité si l'exemple original n'est pas disponible. Par conséquent, la référence démonstrative seule ne permet pas l'identification des contenus comme étant des cas d'un aspect déterminé de l'expérience. Or les capacités conceptuelles, telles que McDowell les défend, devraient déterminer le contenu perçu de telle manière que la reconnaissance d'autres manifestations de ce contenu serait possible. Cependant, en ce qui concerne les contenus raffinés de l'expérience, l'application de la référence démonstrative dépend de l'organisation du contenu perceptif par des règles qui précèdent l'utilisation de ce type de capacité conceptuelle.

De plus, on doit noter (et sur ce point les conceptualistes ont raison) que le caractère raffiné de l'expérience n'est pas un contenu non conceptuel tout court. Il est vrai que ce contenu excède la possession de concepts, et dans ce sens on doit élargir la position conceptualiste, mais il n'est pas suffisant de reconnaître ce qui excède les capacités conceptuelles comme purement non conceptuel. Par exemple, bien qu'on admette qu'il n'y a pas de concepts couvrant tous les aspects raffinés de l'expérience (les tonalités, les formes, les bruits), on doit reconnaître, si l'on suit la position merleau-pontyenne, que ces aspects sont assimilés par des étalons de normalité, de bizarrerie, d'attractivité, etc., c'est-à-dire, par des paramètres culturels qui aident à établir le sens de ce qui est perçu. En effet, ce qui est normalement appelé contenu non conceptuel exprime une préconceptualité civilisationnelle qui n'est pas complètement hors d'une organisation culturelle excédant la simple synthèse neutre et soidisant universelle de stimuli objectifs par des règles de prégnance gestaltiste. Même un citoyen métropolitain ne possède aucun concept pour ordonner tous les bruits d'une autoroute encombrée. Cependant, ces bruits sont non seulement discriminés mais aussi immédiatement discriminés comme désagréables, effrayants, ennuyeux, etc., selon des qualifications culturelles larges qui aident à déterminer le sens de ce qui est entendu.

À notre avis, si l'on veut maintenir le concept de «contenu non conceptuel » tout court, celui-ci ne devrait désigner que les éléments sensibles qui ne figurent pas encore dans le réseau historico-culturel du sujet perceptif. Le contenu non conceptuel serait formé, par exemple, par les bruits du trafic tels que ceux appréhendés les premières fois par un Amazonien, c'est-à-dire le contenu qui ne présente pas encore de sens dans le monde perçu, sens qui, pour se fixer, 
dépend non seulement de quelques règles universelles de ségrégation de phénomènes, mais aussi d'un contexte historico-culturel.

La perception peut, en effet, présenter des contenus non conceptuels dans le sens que nous établissons. Comme nous l'avons souligné, l'activité perceptive ne se limite pas à actualiser quelques habitudes culturelles mais peut engendrer des phénomènes qui justement obligent à modifier ces habitudes. L'exemple d'un changement de contexte culturel entre un natif amazonien et un citoyen métropolitain illustre d'une manière extrême cette autonomie du champ phénoménal par rapport aux restrictions culturelles: des phénomènes qui excèdent complètement le contexte culturel seraient quand même perçus, puisque le champ phénoménal implique une ouverture générale au monde, laquelle saisit ce qui se pose dans sa sphère d'action. Peut-être que, au moins dans les premiers moments de leur présentation, les phénomènes qui excèdent le contexte culturel du sujet perceptif s'organisent seulement selon quelques règles générales de prégnance. Mais au fur et à mesure que l'on s'habitue à ces nouveaux phénomènes, les expectatives, les émotions, les souvenirs, les motivations, etc., de divers aspects de la vie culturelle sédimentent des étalons de discrimination phénoménale: même s'ils ne contrarient pas les règles générales de la prégnance, ils cristallisent quelques paramètres particuliers de leur application. La grande contribution des textes intermédiaires de Merleau-Ponty au débat contemporain sur la perception se trouve dans la mise en relief de ces paramètres particuliers.

\section{Remarque sur le dernier Merleau-Ponty}

Dans ses dernières années, Merleau-Ponty considérait encore que les paramètres d'organisation perceptive ne se réduisent pas à quelques règles universelles, mais se dilatent selon quelques habitudes culturelles ${ }^{25}$. Cependant, il se dévouait dans ces années à établir une ontologie de l'Être brut, c'est-à-dire, de l'être antérieur à n'importe quelle idéalisation de la subjectivité, et qui serait responsable de fonder toute activité subjective. En réalité, Merleau-Ponty cherchait à nouveau la genèse de l'expérience en-deçà de la diversité culturelle, telle que la Phénoménologie de la perception le proposait. Toutefois, dans ce livre, la conscience perceptive jouait le rôle de fond inangural de la connaissance (cf. Php, v). Déjà Merleau-Ponty estimait, à la fin de son œuvre, qu'une couche du monde lui-même contenait les structures sensibles indispensables à l'actuation de la sensibilité. Il faut donc « décrire le visible comme quelque chose qui se réalise à travers l'homme, mais qui n'est nullement anthropologique " (VI, 322). Ainsi, l'expérience perceptive, au moins à son niveau le plus général et primaire, ne serait que l'expression de ces structures sensibles

25. Dans Le visible et l'invisible, Merleau-Ponty reconnaît qu' « il y a une information de la perception par la culture qui permet de dire que la culture est perçue " (Le visible et l'invisible, Paris, Gallimard, Col. Tel, 2001, p. 262. Dorénavant cité comme VI). 
inhérentes à l'Être lui-même. Dans quelques passages, Merleau-Ponty semble esquisser une recherche de ce niveau général de l'activité perceptive. Il se demande dans Le visible et l'invisible: "Comment peut-on revenir de cette perception façonnée par la culture à la perception "brute" ou "sauvage" ?" (VI, 262). Il nous semble que Merleau-Ponty reprendrait le caractère universel de la perception, tel que présenté dans la Phénoménologie de la perception, afin de soutenir que les paramètres préculturels d'organisation perceptive manifestent les structures de l'Être lui-même. Le philosophe n'a pas développé suffisamment ce projet; à cause de sa mort prématurée les propos du Visible et l'invisible sont restés inachevés. En tout cas, il semble que la recherche du niveau informé culturellement ne soit pas, comme dans les années cinquante, le point principal de l'analyse mûre de Merleau-Ponty sur la perception. Nous avons cependant essayé de montrer que cette investigation non seulement justifie le paradigme anthropologique de l'incarnation, mais permet aussi d'élargir le débat contemporain sur l'activité perceptive. 